\title{
INCORPORAÇÃO DE RÉGUA PARA MEDIDA DE PROFUNDIDADE NO PROJETO DO PENETRÔMETRO DE IMPACTO STOLF ${ }^{(1)}$
}

\author{
Rubismar Stolf ${ }^{(2)}$, Jorge Hiroshi Murakami ${ }^{(3)}$, Miguel Angelo Maniero ${ }^{(2)}$, Marcio Roberto \\ Soares $^{(4)} \&$ Luiz Carlos Ferreira da Silva ${ }^{(2)}$
}

RESUMO

\begin{abstract}
O penetrômetro de impacto Stolf, em uso desde o seu lançamento em 1982, é um aparelho para medida da resistência do solo, tipo dinâmico. No projeto original, uma escala centimétrica, de profundidade, foi gravada na própria haste de penetração. $O$ objetivo desse procedimento, na época, foi facilitar o entendimento pelos agricultores e permitir a leitura direta na haste de penetração, além de evitar peças adicionais. $O$ objetivo do presente estudo foi incorporar ao projeto original uma régua em escala milimétrica, a fim de trazer a leitura da profundidade ao nível da visão do operador, evitando o agachamento, e aumentar a precisão da leitura. A utilização do equipamento e o procedimento para posicionar o zero da escala são descritos e esquematizados. Foi realizada comparação entre o equipamento original, com leitura na haste, e o equipamento proposto, com leitura na régua, avaliando-se quanto aos valores da resistência determinados e ao tempo de execução de um perfil de solo. A utilização do equipamento com leitura na régua resultou em um tempo médio $21 \%$ menor (teste t, $1 \%$ ). Além de reduzir o tempo de amostragem, verificou-se a preferência de usuários pelo novo procedimento, em razão do maior conforto ao operador, sendo essa modificação finalmente incorporada ao modelo comercial. Fez-se um histórico do desenvolvimento da tecnologia do penetrômetro de impacto, sendo o presente estudo complementar à primeira modificação no projeto construtivo, que permaneceu inalterado por 30 anos.
\end{abstract}

Termos de indexação: penetrômetro dinâmico de solo, régua de leitura em milímetros, operação, acurácia e custo.

\footnotetext{
(1) Apresentado no XL Congresso Brasileiro de Engenharia Agrícola - CONBEA 2011, Cuiabá - MT, Brasil, 24 a 28 de julho 2011. Recebido para publicação em 21 de novembro de 2011 e aprovado em 03 de julho de 2012.

(2) Professor Associado, Departamento de Recursos Naturais e Proteção Ambiental (DRNPA), Centro de Ciências Agrárias (CCA), Universidade Federal de São Carlos (UFSCar). Rod. Anhanguera, Km 174. CEP 13600-970 Araras (SP). E-mail: rubismar@cca.ufscar.br (www.cca.ufscar.br/ rubismar); miguel@cca.ufscar.br; luizfer@cca.ufscar.br

(3) Engenheiro Mecânico, Depto. Desenvolvimento de Produto, Kamaq - Máquinas e Implementos Agrícolas. Araras (SP). E-mail: jorgehm@kamaq.com.br

(4) Professor Adjunto, DRNPA, CCA, UFSCar. E-mail: mrsoares@cca.ufscar.br
} 


\title{
SUMMARY: INTEGRATION OF RULER TO MEASURE DEPTH INTHE DESIGN OF A STOLF IMPACT PENETROMETER
}

\begin{abstract}
The Stolf impact penetrometer is a dynamic cone penetrometer to measure soil resistance, in use since its release in 1982. In the original design, a centimeter scale to measure penetration depth was engraved on the proper penetration rod. The idea was to make the equipment easier to understand for farmers and to allow direct readings on the penetration rod while also avoiding additional parts. The purpose of this study was to incorporate a millimeter ruler to enable the operator to read the depth at eye level, to avoid squatting and to increase the reading accuracy. The equipment use and reset procedure of the depth reading (zero) were described and illustrated. The two reading types were compared in terms of sampling time, using a randomized block design. A significant difference ( $t$ test, $1 \%$ ) was observed, resulting in $21 \%$ less time spent for readings on the ruler. Besides increasing the reading accuracy and reducing the sampling time, the users preferred the new design, which was finally incorporated into the commercial model. A retrospective of the technical development of the penetrometer is presented; this study represents the first modification of the project, which had remained unaltered for 30 years.
\end{abstract}

Index terms: dynamic soil penetrometer, millimeter ruler, operation, accuracy and cost.

\section{INTRODUÇÃO}

O penetrômetro de impacto, lançado em 1982, tornou-se popular entre os agricultores por meio de publicações técnicas que abordavam aspectos práticos, ou seja, características do equipamento e da operação (Stolf et al., 1983), exemplos de uso em cana-de-açúcar (Stolf \& Faganello, 1983) e em pomares de laranja (Stolf, 1987). Nos trabalhos iniciais adotou-se a unidade prática de resistência do solo, impactos $\mathrm{dm}^{-1}$, comum em engenharia civil. A teoria do equipamento foi apresentada cerca de 10 anos depois, permitindo transformar os dados de resistência em MPa (Stolf, 1991), sendo reproduzida em Stolf et al. (1998, 2005). Com isso, a técnica ganhou novo impulso, tornandose conhecida no meio científico. No lançamento do modelo comercial houve o interesse, tanto da instituição como do fabricante, em oferecer um aparelho de baixo custo, na época 25 vezes menor em relação ao penetrógrafo convencional de mola, que evoluiu, ao longo do tempo, para penetrômetros com célula de carga e registrador digital.

Ambos os penetrômetros, convencional e de impacto, cumprem a finalidade de medir a resistência do solo. Os convencionais são operados por meio de uma pressão contínua sobre o aparelho para penetração; os valores de resistência e profundidade vão sendo registrados automaticamente, necessitando de fonte de energia elétrica e conhecimento maior do operador. O penetrômetro de impacto não apresenta limite quanto à resistência, operando normalmente em solos de alta resistência, e tem preço de mercado várias vezes menor. A consolidação do penetrômetro de impacto Stolf no meio científico pode ser medida pelo seu emprego na atualidade. Verifica-se sua utilização em diversos estudos de mapeamento da resistência do solo, de avaliação da compactação e de controle de tráfego, em estudos sobre variabilidade espacial de propriedades do solo e em estudos sobre manejo de lavouras, pastagens e florestas e recuperação de áreas degradadas, como indicador de qualidade do solo, a exemplo dos seguintes artigos científicos, entre os publicados somente em 2010: Barbosa et al., 2010; Camargo et al., 2010; Cavallini et al., 2010; Correa \& Bento, 2010; Cortez et al., 2010; Ferreira et al., 2010; Grego et al., 2010; Guedes Filho et al., 2010; Lima et al., 2010; Machado et al., 2010; Martins et al., 2010; Medeiros et al., 2010; Mestas et al., 2010; Oliveira et al., 2010; Pedron et al., 2010; Portugal et al., 2010; Ramos et al., 2010; Roboredo et al., 2010; Roque et al., 2010; Sanchez-Saenz, 2010; Silva Filho et al., 2010; Silva Junior et al., 2010a,b, Silva et al., 2010; Silveira et al., 2010; Siqueira et al., 2010; Souza et al., 2010; Tablada et al., 2010; Tavares Filho et al., 2010; Vieira et al., 2010.

No projeto original, a escala de profundidade foi gravada na própria haste de penetração, a fim de facilitar a compreensão pelos agricultores do funcionamento do aparelho, ainda desconhecido por eles, e dispensar peças adicionais. Contudo, o fato de a escala estar gravada na própria haste obriga um dos operadores a realizar a leitura agachado, enquanto outro faz as anotações. Além da dificuldade de leitura, a escala gravada na haste é centimétrica, pela impossibilidade de se gravar uma escala milimétrica, o que permitiria maior precisão de leitura.

O objetivo deste estudo foi incorporar ao projeto original uma régua de leitura para proporcionar conforto, permitindo leituras de profundidade ao nível da visão do operador, aumentar a precisão das leituras, adotando a escala milimétrica, e reduzir o tempo de execução das medidas. 


\section{MATERIAL E MÉTODOS}

\section{Desenvolvimento do protótipo e testes realizados}

Desenvolveu-se um protótipo da régua de medida de profundidade utilizando material plástico tubular acoplado ao penetrômetro (Figura 1a). Inseriu-se o protótipo na rotina de uso de determinação da resistência do solo na área agrícola da Destilaria Londra (Itaí - SP). Como a citada empresa já utilizava o equipamento anterior, com o sistema de leitura na haste, foi possível observar a preferência dos operadores pelo protótipo, abandonando o sistema anterior. Observações adicionais, como durabilidade, facilidade para montagem e tamanho para transporte, serviram de base para a construção do modelo definitivo em colaboração com a empresa de máquinas e implementos agrícolas, fabricante do penetrômetro de impacto (Figura 1b,c). Utilizou-se alumínio tubular, gravando-se a escala milimétrica, 0 a $70 \mathrm{~cm}$, em baixo relevo no tubo, utilizando-se a técnica de corrosão por meio ácido. Os aspectos construtivos são apresentados no item Resultados.

Para teste do tempo de execução de leitura e dos valores de resistência, foram utilizados dois tratamentos com sete repetições: Tratamento 1 penetrômetro de impacto com escala de profundidade gravada na haste de penetração (haste); e Tratamento 2 - penetrômetro de impacto com a régua de medida proposta (régua).

Avaliou-se o tempo de execução de uma amostra em profundidade, até atingir ou ultrapassar $50 \mathrm{~cm}$. A determinação do tempo iniciou-se após o posicionamento do aparelho na superfície do terreno e terminou após a retirada do aparelho do solo, não se
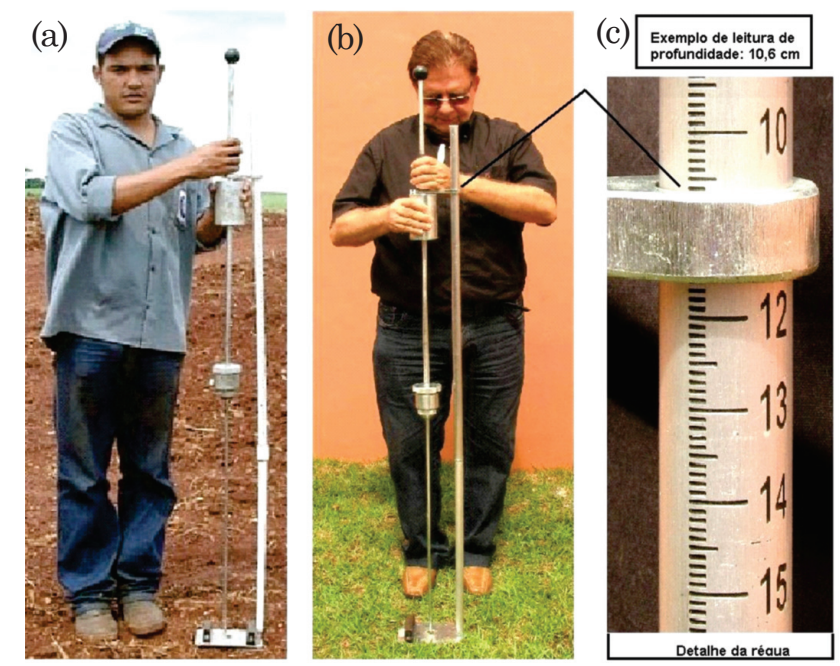

Figura 1. Detalhe do equipamento em teste, indicando: (a) a régua no protótipo em plástico; (b) o modelo definitivo de alumínio; c) o detalhe do nível de leitura do modelo definitivo. computando o tempo de deslocamento entre pontos de amostragem. $\mathrm{O}$ ensaio foi realizado no campus de Araras da UFSCar, em área de Latossolo Vermelho, após rotação cana-de-açúcar-milho-crotalária, apresentando resistência média de $2,53 \mathrm{MPa}$ na profundidade de $0-50 \mathrm{~cm}$.

Os dados de resistência obtidos na amostragem foram equalizados por um programa computacional (Stolf, 2011), em camadas de $5 \mathrm{~cm}$. Aplicou-se o teste t para comparação das médias dos tratamentos (haste $\mathrm{x}$ régua) para: (a) resistência nas várias profundidades e média geral dos perfis; e (b) tempo de execução do perfil.

\section{RESULTADOS}

\section{Montagem e calibração do modelo penetrômetro com régua desenvolvido}

A figura 2 ilustra o primeiro sistema, antigo, para leitura na própria haste de penetração; na figura 3 encontra-se a nova versão proposta, com régua. Adotouse o modelo tubular de régua, para permitir maior ângulo de visão da escala de leitura, e o alumínio, por se tratar de um material leve, de baixa densidade e resistente à oxidação.

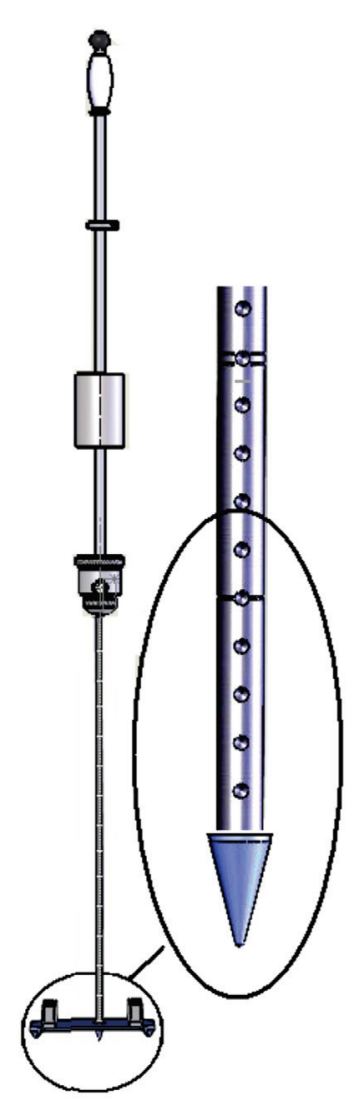

Figura 2. Método anterior: medida da profundidade na própria haste de penetração. 
Para montagem, a régua é parafusada na chapa de fixação e nivelamento da superfície do solo (Figura $3 a)$, até atingir cerca de $1 \mathrm{~cm}$ de altura. Na parte superior, a régua apoia-se dentro da argola de leitura (Figura 3b). Para calibração do zero, a base do cone de penetração deverá facear o plano inferior da placa de fixação (Figura 3a), enquanto o zero da régua deverá facear o plano superior da argola de leitura (Figura 3b). Os novos modelos de penetrômetro de impacto já são construídos de forma que, ao parafusar a régua até a metade, ou seja, faltando $1 \mathrm{~cm}$ para atingir o final da rosca (Figura 3a), o aparelho esteja aproximadamente zerado para o uso (Figura 3b). Um ajuste fino poderá ser realizado parafusando ou desparafusando a régua (Figura 3a), ou seja, subindo ou descendo um pouco a régua. A calibração do zero não necessariamente deve ser feita na posição vertical, sendo mais fácil fazê-la repousando o aparelho na horizontal.

O comprimento da régua é de 1,44 $\mathrm{m}$. Ela foi dividida em duas partes: para transporte e uso no campo. Projetou-se uma conexão rápida entre as duas partes, através de imã. Para se deslocar no campo não é necessário desparafusar a parte inferior da régua; basta puxar a parte superior, desconectando-a.

O próprio usuário que já possui o penetrômetro poderá realizar a modificação ou fazê-la por intermédio do fabricante, sendo o projeto desenhado para minimizar as alterações. Assim, o limitador superior da massa de impacto do modelo anterior passa também à função de sustentação e nível de medida; a placa de

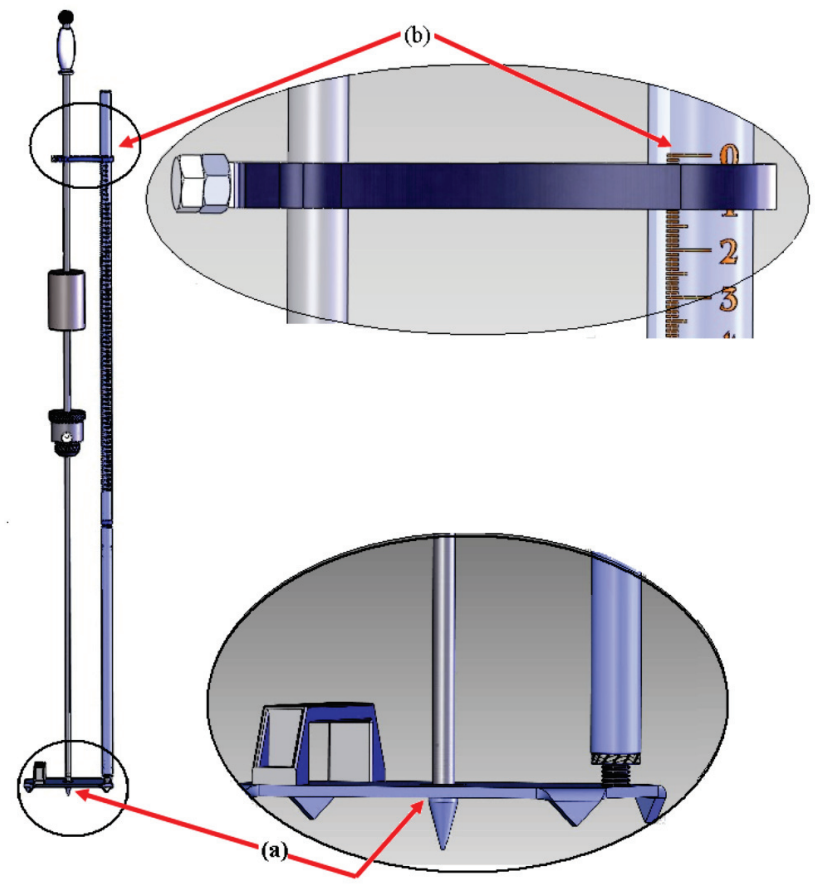

Figura 3. Método atual: medida da profundidade na régua. Posições (a) e (b) para ajustar o zero. nivelamento recebe um parafuso para sustentar a régua na parte inferior. Na figura 4 encontra-se o desenho explodido do penetrômetro de impacto, com todos os seus componentes.

\section{Testes de tempo de execução de levantamento de resistência do solo}

Os dados de resistência do solo e de tempo de execução obtidos nos testes são apresentados no quadro 1. Quanto à resistência do solo, não foram detectadas diferenças significativas entre os tratamentos a cada profundidade, bem como na média geral, sendo o valor médio para o tratamento com leitura na haste de 2,50 $\mathrm{MPa}$ e, na régua, de 2,56 MPa. Mesmo sendo, por princípio, a escala milimétrica mais precisa que a centimétrica, os dados mostram que no caso específico o aumento da precisão não era necessário. Quanto ao tempo de execução, verificou-se diferença significativa $(p<0,01)$, a $1 \%$, resultando em redução de $21 \%$ no tempo quando se utilizou a medida com leitura na régua, em relação à leitura na haste (Quadro 1).

Os usuários por empréstimo, tanto no período do uso do protótipo como no do modelo definitivo, optaram pelo uso da régua. Além da maior precisão da escala, a proximidade da visão em relação à régua e a leitura em pé, evitando o desconforto em relação à posição agachada, foram fatores determinantes.

Os resultados, independentemente dos tratamentos, ou seja, da forma de leitura, evidenciam duas características do atributo resistência do solo: a de obtenção de uma quantidade grande de informações em tempo relativamente curto, comparativamente aos demais atributos para estudos de compactação do solo, e de dispensar complementação laboratorial. O tempo

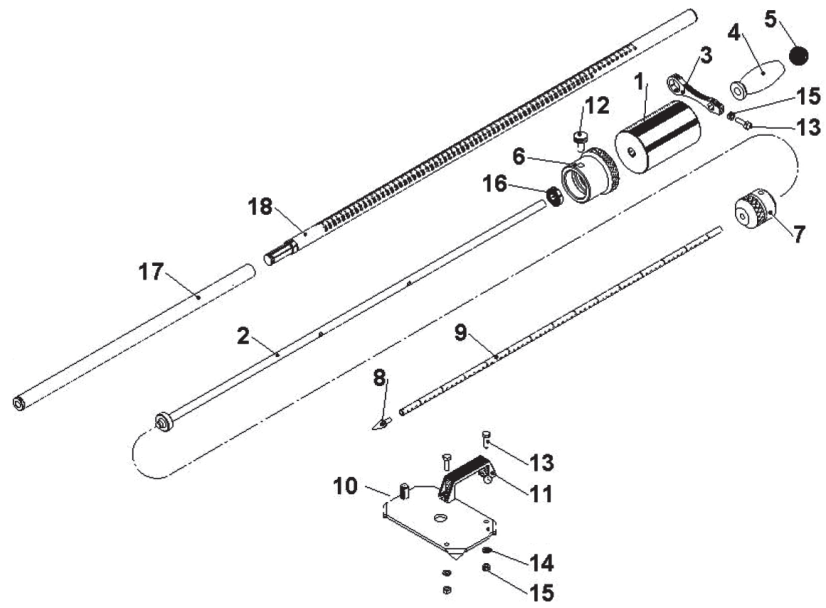

Figura 4. Catálogo de peças, contendo 18 componentes do penetrômetro de impacto. Obs.: Modificações: argola de nível de leitura (3); inclusão, na chapa (10) de nivelamento, de um parafuso para fixar a base da régua (17), que por sua vez se conecta à régua propriamente dita através de imã (18). 
de execução de um perfil até $50 \mathrm{~cm}$, na média dos tratamentos, resultou em cerca de 1 min. Em levantamentos, em grandes áreas agrícolas, o tempo de execução é muito pequeno comparativamente a outros fatores, como: preparação do material, deslocamento para os pontos de amostragem, seleção do ponto específico no local, colocação da placa de nivelamento e posicionamento do aparelho para leitura. Portanto, a redução de tempo total de amostragem em um método já ágil por natureza deve focar o planejamento dos pontos amostrais e a logística de deslocamento no campo e não a redução do tempo de leitura dos dados propriamente dita. Recomenda-se, aos operadores, a utilização de um tempo suficiente para a realização de leituras cuidadosas e não se ater à velocidade de execução.
Considerando que os levantamentos de resistência do solo geram um grande volume de dados, é interessante dispor de programas computacionais como o utilizado para confecção do quadro 1 e da figura 5 (Stolf, 2011).

\section{CONCLUSÃO}

A régua de medida com escala em milímetros incorporada ao penetrômetro reduziu o tempo de leitura; proporcionou maior conforto ao operador, evitando a posição agachada; e facilitou a atividade, com a aproximação da leitura de profundidade ao nível da visão deste.

Quadro 1. Resistência do solo $(0-50 \mathrm{~cm})$ e respectivo tempo de execução do perfil e resultados estatísticos da comparação de médias para diferentes tratamentos

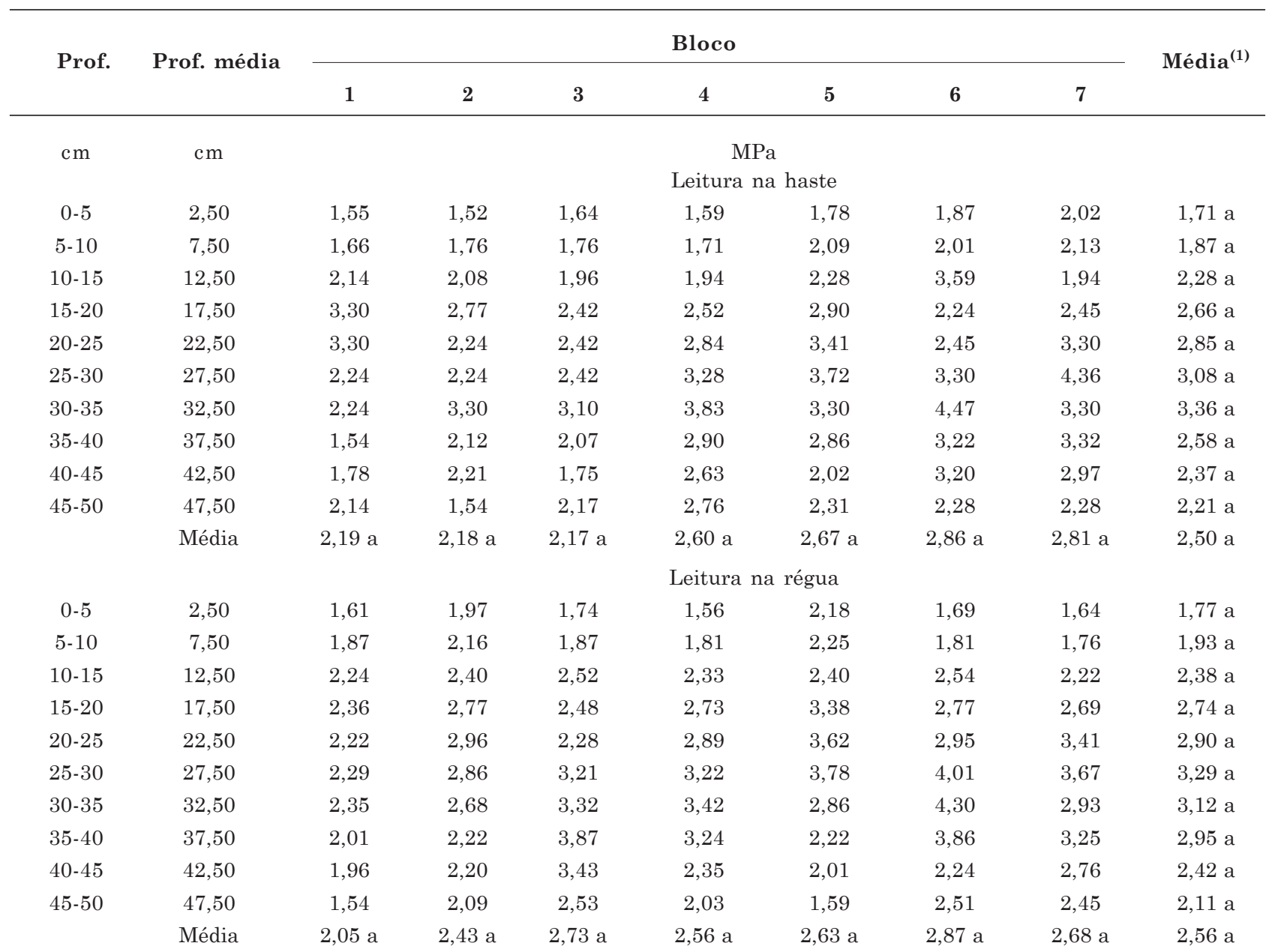

Tempo de execução da amostra de um perfil até $50 \mathrm{~cm}$ de profundidade (em segundos)

$\begin{array}{lllllllll}\text { Leitura na haste } & 69 & 60 & 59 & 69 & 62 & 62 & 55 & 62 \mathrm{a} \\ \text { Leitura na régua } & 47 & 51 & 42 & 48 & 55 & 55 & 45 & 49 \mathrm{~b}\end{array}$

${ }^{(1)}$ Médias seguidas de mesma letra não diferem entre si. Médias seguidas de letras diferentes diferem significativamente a $1 \%$, segundo o teste t. Obs.: as comparações de médias são todas entre tratamentos; não há comparações dentro de tratamento. 


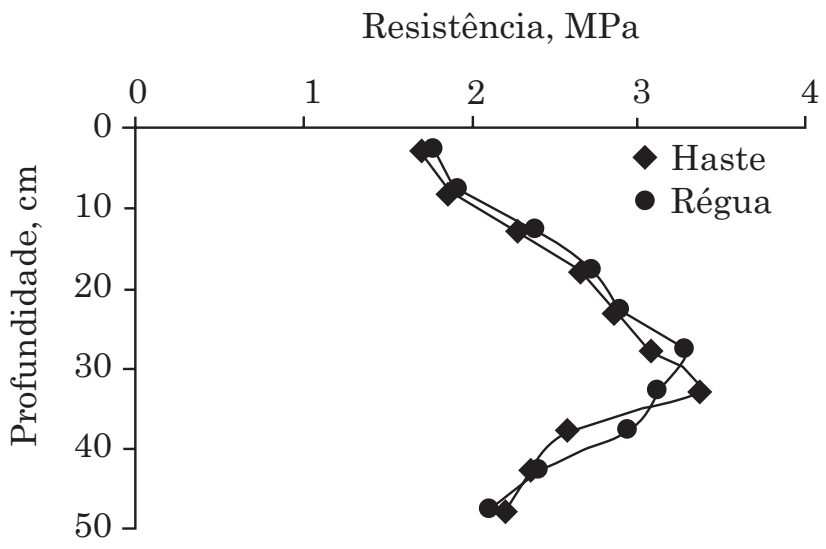

Figura 5. Média dos perfis de resistência do solo, com leitura na haste e na régua.

\section{AGRADECIMENTOS}

A Renato Carretin, Kamaq, pela oportunidade de discussões e sugestões; aos alunos da UFSCar, de graduação, Leandro Marcussi e Rafael Dreux Miranda Fernandes, e de pós-graduação, Túlio Caio Binotti, pela colaboração nas atividades de campo; e a Elpidio Storolli, jornalista, pelas fotografias.

\section{LITERATURA CITADA}

BARBOSA, C.; CUNHA, C.M.L.; CARVALHO, P.F. \& MATHIAS. D.T. Técnica de avaliação das características hidrogeomorfológicas de vertentes para o planejamento urbano. R. Geogr. S8, 2010.

CAMARGO, L.A.; MARQUES JUNIOR, J. \& PEREIRA, G.T. Spatial variability of physical attributes of an alfisol under different hillslope curvatures. R. Bras. Ci. Solo, 34:617$630,2010$.

CAVALLINI, M.C.; ANDREOTTI M.; OLIVEIRA L.L.; PARIZ C.M. \& CARVALHO M.P. Relações entre produtividade de Brachiaria brizantha e atributos físicos de um Latossolo do cerrado. R. Bras. Ci. Solo, 34:1007-1015, 2010.

CORREA, R.S. \& BENTO, M.A.B. Qualidade do substrato minerado de uma área de empréstimo revegetada no distrito federal. R. Bras. Ci. Solo, 34:1435-1443, 2010.

CORTEZ, J.W.; FERNANDES, A.L.T.; CARVALHO FILHO, A.; SILVA, R.P. \& FURLANI, C.E.A. Modificação dos parâmetros físico-hídricos do solo sob diferentes sistemas de irrigação na cafeicultura. R. Bras. Ci. Agr., 5:244-249, 2010.

FERREIRA, W.C.; BOTELHO S.A.; DAVIDE A.C.; FARIA, J.M.R. \& FERREIRA, D.F. Regeneração natural como indicador de recuperação de área degradada a jusante da usina hidrelétrica de Camargos, MG. R. Árvore, 34:651$660,2010$.
GREGO, C.R.; VIEIRA, S.R. \& XAVIER, M.A. Spatial variability of some biometric attributes of sugarcane plants (variety IACSP93-3046) and its relation to physical and chemical soil attributes. Bragantia, 69:107-119, 2010.

GUEDES FILHO, O.; VIEIRA, S.R.; CHIBA, M.K.; NAGUMO, C.H. \& DECHEN, S.C.F. Spatial and temporal variability of crop yield and some Rhodic Hapludox properties under no-tillage. R. Bras. Ci. Solo, 34:1-14, 2010.

LIMA, C.G.R.; CARVALHO, M.P.; NARIMATSU, K.C.P.; SILVA, M.G. \& QUEIROZ, H.A. Atributos físico-químicos de um Latossolo do Cerrado brasileiro e sua relação com características dendrométricas do eucalipto. R. Bras. Ci. Solo, 34:163-173, 2010

MACHADO, W.; TELlES, T.S.; TAVARES FILHO, J.; GUIMARÃES, M.F.; ALVES, G.B. \& BORGES, J.L.B. Physical properties of a Rhodic Haplustox under two sugarcane harvesting systems. R. Bras. Ci. Solo, 34:18031809, 2010.

MARTINS, C.A.S.; PANDOLFI, F.; PASSOS, R.R.; REIS, E.F. \& CABRAL, M.B.G. Avaliação da compactação de um Latossolo Vermelho-Amarelo sob diferentes coberturas vegetais. Biosci. J., 26:79-83, 2010.

MEdeiros, J.C.; FIGUEIREDO, G.C. \& MAFRA, A.L. Portable penetrometer for agricultural soil: sensitivity test to identify critical compaction depth. R. Bras. Ci. Solo, $34: 1823-1829,2010$

MESTAS, R.M., ROQUE, M.W., MATSURA, E.E.; BIZARY, D.R. \& PAZ, A. Variabilidad espacial de los atributos físicohídricos del suelo y de la productividad del cultivo de fréjol (Phaseolus vulgaris L) irrigado bajo un sistema de siembra directa. R. Ci. Agr., 33:307-313, 2010.

OLIVEIRA. C.A.; KLIEMANN. H.J.; CORRECHEL. V. \& SANTOS, F.C.V. Avaliação da retenção de sedimentos pela vegetação ripária pela caracterização morfológica e físico-química do solo. R. Bras. Eng. Agríc. Amb., 14, 12811287, 2010.

PEDRON, F.A.; FINK, J.R.; DALMOLIN, R.S.D. \& AZEVEDO, A.C. Morfologia dos contatos entre solo-saprolito-rocha em neossolos derivados de arenitos da formação caturrita no Rio Grande do Sul. R. Bras. Ci. Solo, 34:1941-1950, 2010 .

PORTUGAL, A.F.; CostA, O.D.V. \& CostA, L.M. Propriedades físicas e químicas do solo em áreas com sistemas produtivos e mata na região da Zona da Mata mineira. R. Bras. Ci. Solo, 34:575-585, 2010.

RAMOS, F.T.; MONAR, Y.C.; NUNES, M.C.M.; CAMPOS, D.T.S. \& RAMOS, D.T. Indicadores de qualidade em um latossolo vermelho-amarelo sob pastagem extensiva no pantanal matogrossense. R. Caatinga, 23:112-120, 2010.

ROBOREDO, D.; MAIA, J.C.S.; OLIVEIRA, O. \& ROQUE, C.G. Uso de dois penetrômetros na avaliação da resistência mecânica de um Latossolo Vermelho distrófico. Eng. Agríc., 30:308-314, 2010.

ROQUE, A.A.O.; SOUZA, Z.M.; BARBOSA, R.S. \& SOUZA, G.S. Controle de tráfego agrícola e atributos físicos do solo em área cultivada com cana-de-açúcar. Pesq. Agropec. Bras., 45:744-750, 2010. 
SANCHEZ-SAENZ, C.M.; SOUZA, Z.M.; MATSURA, E.E. \& FREITAS, N.R.S. Efecto de la cobertura en las propiedades del suelo y en la producción de fríjol irrigado. Rev. U.D.C.A. Actual. Divulg .Cient., 13:41-50, 2010.

SILVA FILHO, E.P.; COTTAS L.R. \& MARINI, G.B.S. Avaliação da compactação dos solos em áreas de pastagens e florestas em Porto Velho-Rondônia. B. Geogr., 28:145-155, 2010.

SILVA JUNIOR, C.A.; MEURER, I.; CARVALHO, L.A.; SILVA, M.A.C. \& SOUZA JUNIOR, W.S. Soil physical quality related to some methods of tillage in deployment of sugarcane. In: CONGRESS OF THE INTERNATIONAL SOIL CONSERVATION ORGANIZATION, 16., Santiago, 2010. Anais... Santiago, Universidad de Chile, 2010a. p.127-128.

SILVA JUNIOR, C.A.; CARVALHO, L.A.; MEURER, I.; LIBARDI, P.L.; SILVA, M.A.C. \& OLIVEIRA, E.C.A. Alterações nos atributos físicos de um Latossolo Vermelho sob diferentes métodos de preparo para o plantio da canade-açúcar. R. Agrar., 3:111-118, 2010 b.

SILVA, W.M.; CREMON, C.; MAPELI, N.C.; TIEPPO, R.C.: CARVALHO, J.M.; LONGO, L.; FERRI, M. \& MAGALHÃES, W.A. Emissão de $\mathrm{CO}_{2}$ e potencial de conservação de $\mathrm{C}$ em solo submetido à aplicação de diferentes adubos orgânicos. R. Agrar., 3:34-43, 2010.

SILVEIRA, D.C.; MELO FILHO, J.F.; SACRAMENTO, J.A.A.S. \& SILVEIRA, E.C.P. Relação umidade versus resistência à penetração para um Argissolo Amarelo distrocoeso no recôncavo da Bahia. R. Bras. Ci. Solo, 34:659-667, 2010.

SIQUEIRA, D.S.; MARQUES, J. \& PEREIRA, G.T. The use of landforms to predict the variability of soil and orange attributes. Geoderma, 155:55-66, 2010.

SOUZA, Z.M.; MARQUES JUNIOR, J. \& PEREIRA, G.T. Geoestatística e atributos do solo em áreas cultivadas com cana-de-açúcar. Ci. Rural, 40:48-56, 2010.

STOLF, R. \& FAGANELLO, B.F. Utilização do penetrômetro de impacto, modelo IAA/Planalsucar-Stolf, na recomendação do preparo do solo na Usina Nossa Senhora Aparecida (Pontal-SP). STAB, 1:11-22, 1983.
STOLF, R.; FERNADES, J. \& FURLANI NETO, V.L. Penetrômetro de impacto modelo IAA/Planalsucar-Stolf: recomendação para seu uso. STAB, 1:18-23, 1983.

STOLF, R. A compactação do solo e perspectivas de subsolagem em citrus. Laranja, 2:283-308, 1987.

STOLF, R. Teoria e teste experimental de fórmulas de transformação dos dados de penetrômetro de impacto em resistência do solo, R. Bras. Ci. Solo, 15:229-235, 1991.

STOLF, R.; CASSEL, D.K.; KING, L.D. \& REICHARDT, K. Measuring mechanical impedance in clayey gravelly soils. R. Bras. Ci. Solo, 22:189-196, 1998.

STOLF, R., REICHARDT, K. \& VAZ, C.P.M. Response to "Comments on 'simultaneous measurement of soil penetration resistance and water content with a combined penetrometer-TDR moisture probe' and 'a dynamic cone penetrometer for measuring soil penetration resistance"'. Soil Sci Soc. Am. J., 69:927-929, 2005.

STOLF, R. Penetrômetro de Impacto Stolf- programa de manipulação de dados em Excel-VBA. UFSCar, 2011. Disponível em: <http://www.cca.ufscar.br/drnpa/ hprubismar.htm>, item 100. Acesso em: 16 nov. de 2011.

TABLADA, R.N.; CRUZ, V.L.; PALÁCIO, S.R. \& GONZÁLEZ, A.P. Estudio de la resistencia de penetración de un suelo Vertisol con el uso del penetrómetro de impacto en la localidad de "Antonio Maceo" Cuba. Ci. Holguín, 16:1-9, 2010. Disponível em: <http://cienciahlg.idict.cu/index.php/ cienciasholguin/article/view/539>.

TAVARES FILHO, J.; BARBOSA, G.M.C. \& RIBON, A.A. Physical properties of dystrophic Red Latosol (Oxisol) under different agricultural uses. R. Bras. Ci. Solo, 34:925933, 2010.

VIEIRA, S.R.; PIERRE, L.H.; GREGO, C.R.; SIQUEIRA, G.M. \& DAFONTE, J.D. A Geostatistical analysis of rubber tree growth characteristics and soil physical attributes. In: ATKINSONAND, P.M. \& LLOYD, C.D., eds. GeoENV VII - Geostatistics for environmental applications. Amsterdam, Springer, 2010. p.255-264. 